\title{
Millimeter interferometric observations of FU Orionis-type objects in Cygnus
}

\author{
Á. Kóspál ${ }^{\star}$ \\ Leiden Observatory, Leiden University, PO Box 9513, 2300 RA, Leiden, The Netherlands \\ e-mail: kospal@strw.leidenuniv.nl
}

Received 29 August 2011 / Accepted 16 September 2011

\begin{abstract}
Context. FU Orionis-type objects (FUors) are low-mass young eruptive stars that probably represent an evolutionary phase characterized by episodic periods of increased accretion rate from the circumstellar disk to the star. Theory predicts that a circumstellar envelope, the source of continuous mass infall onto the disk, is necessary for triggering such accretion bursts.

Aims. We intend to study the spatial and velocity structure of circumstellar envelopes around FUors by means of molecular line observations at millimeter wavelengths. We target three prototypical FUors, as well as an object possibly in a pre-outburst state.

Methods. We present archival PdBI interferometric observations of the $J=1-0$ line of ${ }^{13} \mathrm{CO}$ at $110.2 \mathrm{GHz}$. For three of our targets, these represent the first millimeter interferometric observations. The data allow study of the molecular environment of the objects with a spatial resolution of a thousand $\mathrm{AU}$ and a velocity resolution of $0.2 \mathrm{~km} \mathrm{~s}^{-1}$.

Results. Strong, narrow ${ }^{13} \mathrm{CO}(1-0)$ line emission is detected from all sources. The emission is spatially resolved in all cases, with deconvolved sizes of a few thousand AUs. For V1057 Cyg and V1331 Cyg, the emitting area is rather compact, suggesting that the origin of the emission is a circumstellar envelope surrounding the central star. For V1735 Cyg, the ${ }^{13} \mathrm{CO}$ emission is offset from the stellar position, indicating that the source of this emission may be a small foreground cloud, also responsible for the high reddening of the central star. The ${ }^{13} \mathrm{CO}$ emission towards V1515 Cyg is the most extended in the sample, and it apparently coincides with the ring-like optical reflection nebula associated with V1515 Cyg.

Conclusions. We suggest that millimeter interferometric observations are indispensable for a complete understanding of the circumstellar environment of FUors. Any theory of the FUor phenomenon that interprets the geometry of the circumstellar structure and its evolution using single-beam measurements must be checked and compared to interferometric observations in the future.
\end{abstract}

Key words. circumstellar matter - stars: formation - infrared: stars

\section{Introduction}

FU Orionis-type objects, or FUors, constitute a small group of young stars characterized by large outbursts in visible light, attributed to highly enhanced accretion (Hartmann \& Kenyon 1996). During these outbursts, accretion rates from the circumstellar disk to the star are on the order of $10^{-4} M_{\odot} / \mathrm{yr}$, three orders of magnitude higher than in quiescence. Enhanced accretion is often accompanied by enhanced mass loss, because most FUors have optical jets, molecular outflows, and optically visible ringlike structures that are sometimes explained by expanding shells thrown off during previous outbursts.

During a single, century-long outburst, as much as $0.01 M_{\odot}$ of the material can be dumped onto the stellar surface. Thus, the inner disk needs to be replenished after each outburst, possibly by material from the infalling envelope. Recent theoretical studies show that the continuous infall from the envelope is also needed to trigger the outbursts (Vorobyov \& Basu 2006). After many outbursts, the envelope vanishes, and the object finally enters a state of permanently low accretion. This general paradigm of the evolution of young, lowmass stars was invoked by Quanz et al. (2007) to explain the observed diversity of FUors: while some objects are still

* Current address: Research and Scientific Support Department, European Space Agency, PO Box 299, 2200 AG, Noordwijk, The Netherlands. deeply embedded (e.g. L1551 IRS 5, V1735 Cyg), others have already cleared away part of their envelopes (e.g. V1057 Cyg, V1515 Cyg). Statistics show that probably all low-mass young stars undergo FUor-like phases during their evolution, implying that FUors might be the clue objects that can lead to understanding envelope evolution and dispersal.

Interferometric observations of molecular line emission have been successfully used to probe the small-scale structure of molecular material around young stellar objects (YSOs). For example, for a sample of low-mass YSOs in Taurus (Hogerheijde et al. 1998), Serpens (Hogerheijde et al. 1999), and Ophiuchus (van Kempen et al. 2009), it was found that most YSOs are surrounded by compact envelopes of up to a few thousand $\mathrm{AU}$ in radius, which are well traced by ${ }^{13} \mathrm{CO}$ and $\mathrm{C}^{18} \mathrm{O}$. Condensations, inhomogeneities in the envelopes can be seen in $\mathrm{HCO}^{+}$and ${ }^{13} \mathrm{CO}$. The $\mathrm{HCO}^{+}$and $\mathrm{HCN}$ molecules trace the walls of the outflow, while $\mathrm{SiO}$ and $\mathrm{SO}$ emission originates in shocked material in the outflow. These observations provided detailed kinematical picture of the envelopes (for molecular studies of some individual YSOs, see e.g. Jørgensen et al. 2004; Matthews et al. 2006; Brinch et al. 2009).

Despite being "accretion laboratories", there are relatively few molecular gas observations published for FUors. Singledish observations often show strong $\mathrm{CO}$ emission towards FUors, and line profiles sometimes indicate molecular outflows (e.g. Hartmann \& Kenyon 1996, and references therein). 
However, due to the large single-dish beam, it is not possible to study the spatial distribution of the emission using these data. Out of the nine FUors listed in Hartmann \& Kenyon (1996), millimeter interferometric observations are only available for L1551 IRS 5 (e.g. Momose et al. 1998).

In this paper, we present interferometric observations of the molecular emission from four FUor-type objects in Cygnus: V1057 Cyg, V1331 Cyg, V1515 Cyg, and V1735 Cyg. With the exception of V1331 Cyg (Levreault 1988; McMuldroch et al. 1993), these data represent the first millimeter interferometric observations for our targets. We use the ${ }^{13} \mathrm{CO}(1-0)$ line emission to perform a high spatial and spectral resolution study of the gaseous material around FUors. We analyze the spatial and kinematic structure of the ${ }^{13} \mathrm{CO}$ gas, and check whether the emission can be associated with the circumstellar envelopes. Our results can be compared with those obtained for normal YSOs. Such a comparison may also contribute to the ongoing debate over whether all young stars undergo an eruptive phase in their early evolution or FUors are atypical objects.

\section{Observations}

We reduced unpublished ${ }^{13} \mathrm{CO}$ observations of our targets obtained with the Plateau de Bure Interferometer (PdBI) on May 30 and 31, 1993 (program ID: C057, PI: Fiebig). The observations were carried out in snapshot mode (about $1 \mathrm{~h}$ of onsource correlation time per object), using four antennas (4D1 configuration), and the baselines ranged from 24 to $64 \mathrm{~m}$. The receiver was tuned to the ${ }^{13} \mathrm{CO}(1-0)$ line at $110.2 \mathrm{GHz}$ (lower sideband), the channel spacing was $78 \mathrm{kHz}\left(0.21 \mathrm{~km} \mathrm{~s}^{-1}\right)$. At this wavelength, the single-dish HPBW is $45^{\prime \prime}$. Bright quasars $(3 C 454.3,2005+403,2037+511,2200+420,0648-165$, and 0727-115) were observed to enable RF bandpass, phase, and amplitude calibration. The phase center was $20^{\mathrm{h}} 58^{\mathrm{m}} 53^{\mathrm{s}} .7$ $+44^{\circ} 15^{\prime} 27^{\prime \prime} .9$ for V1057 Cyg, $21^{\mathrm{h}} 01^{\mathrm{m}} 09^{\mathrm{s}} .3+50^{\circ} 21^{\prime} 42^{\prime \prime} .2$ for V1331 Cyg, $20^{\mathrm{h}} 23^{\mathrm{m}} 47^{\mathrm{s}} .9+42^{\circ} 12^{\prime} 24^{\prime \prime} .8$ for V1515Cyg, and $21^{\mathrm{h}} 47^{\mathrm{m}} 20^{\mathrm{s}} .7+47^{\circ} 32^{\prime} 04^{\prime \prime} .1$ for V1735 Cyg.

Data reduction was done in the standard way with CLIC, a GILDAS-based application written especially for reducing PdBI data ${ }^{1}$. The rms phase noise was less than $18^{\circ}$ for all observations. We estimate a flux calibration accuracy of $\approx 15 \%$. MAPPING was used to create naturally weighted dirty maps in an area of $64^{\prime \prime} \times 64^{\prime \prime}$, which were then deconvolved using the Clark CLEAN method. The synthesized beam is approximately $7^{\prime \prime} \times 6^{\prime \prime}$, while the rms noise in the channel maps is $\approx 0.15 \mathrm{Jy} /$ beam. For the exact beam parameters, see the log of observations in Table 1.

\section{Results}

The ${ }^{13} \mathrm{CO}(1-0)$ spectra of our sources, integrated over the whole $64^{\prime \prime} \times 64^{\prime \prime}$ maps, are plotted in Fig. 1. Strong ${ }^{13} \mathrm{CO}(1-$ $0)$ emission was detected towards all sources with high signalto-noise ratio. In all cases, we found an emission line coinciding with the systemic velocity of each source (from optical and near-IR spectra, Herbig 1977, Kenyon \& Hartmann 1989, and Chavarría et al. 1979 determined heliocentric radial velocities of $-14 \pm 2,-15 \pm 2,-12 \pm 2$, and $-10 \pm 2 \mathrm{~km} \mathrm{~s}^{-1}$, corresponding to LSR velocities of $1.9,0.6,5.2$, and $3.9 \mathrm{~km} \mathrm{~s}^{-1}$ for V1057 Cyg, V1331 Cyg, V1515 Cyg, and V1735 Cyg, respectively). We collapsed a few channels centered on the peak emission, calculated visibility amplitudes as a function of $u v$ radius (Fig. 2),

\footnotetext{
${ }^{1}$ http://www.iram.fr/IRAMFR/GILDAS
}
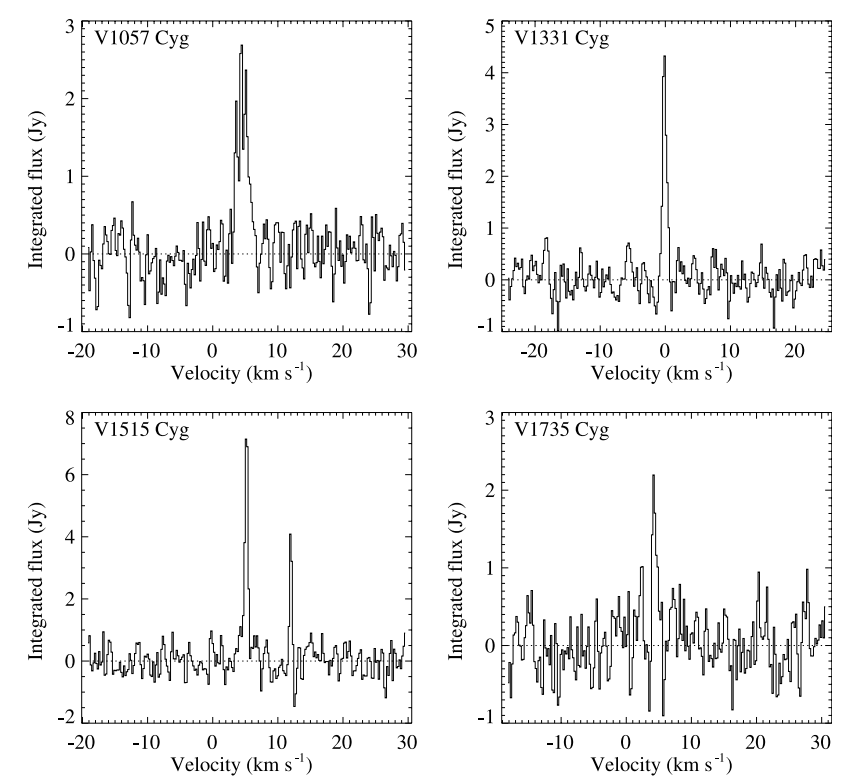

Fig. 1. ${ }^{13} \mathrm{CO}(1-0)$ spectra of our targets obtained with the PdBI. Fluxes were integrated over an area of $64^{\prime \prime} \times 64^{\prime \prime}$ centered on the phase center.

and produced velocity-integrated maps (Fig. 3). The LSR velocities where the emission peaks are listed in Table 1. Velocityintegrated line fluxes calculated for the emission areas visible in Fig. 3 are also listed in Table 1.

\subsection{Line profiles}

The line profile of V1057 Cyg is the broadest in our sample $\left(\mathrm{FWHM} \approx 2.1 \mathrm{~km} \mathrm{~s}^{-1}\right.$ ), and is centered at $4.6 \mathrm{~km} \mathrm{~s}^{-1}$. This is consistent with the velocity seen in single-dish ${ }^{12} \mathrm{CO}(1-0)$, ${ }^{12} \mathrm{CO}(2-1)$, and ${ }^{13} \mathrm{CO}(1-0)$ data by Bechis \& Lo (1975) and Levreault (1988). V1331 Cyg shows a narrow ${ }^{13} \mathrm{CO}(1-0)$ line $\left(\mathrm{FWHM} \approx 0.7 \mathrm{~km} \mathrm{~s}^{-1}\right.$ ). McMuldroch et al. (1993) presents Owens Valley interferometric observations of V1331 Cyg in the same line, as well as in other transitions and other isotopes of CO. The velocities of these lines are consistent with ours. V1515 Cyg shows two separate, narrow emission components $\left(\mathrm{FWHM} \approx 0.6 \mathrm{~km} \mathrm{~s}^{-1}\right.$ ) at $5.1 \mathrm{~km} \mathrm{~s}^{-1}$ and at $11.9 \mathrm{~km} \mathrm{~s}^{-1}$. Both of these components are also visible in single-dish ${ }^{12} \mathrm{CO}(3-2)$ and ${ }^{13} \mathrm{CO}(2-1)$ data from Evans et al. (1994). The spectrum of V1735 Cyg shows a narrow, single-peaked ${ }^{13} \mathrm{CO}(1-0)$ line $\left(\mathrm{FWHM} \approx 0.7 \mathrm{~km} \mathrm{~s}^{-1}\right.$ ). The shape and position of this line coincides well with that of the ${ }^{13} \mathrm{CO}(2-1)$ line detected in singledish data by Evans et al. (1994). The ${ }^{12} \mathrm{CO}(2-1)$ line from the same paper shows self-absorption at this velocity, and broad line wings indicating outflow activity. We plotted position-velocity diagrams along different angles through our sources, but except for V1515 Cyg (which will be discussed later), we found no significant velocity gradients.

\subsection{Spatial extent}

In Fig. 2 we plotted the visibility amplitudes as a function of $u v$ radius. Since these are snapshot observations, the $u v$-space is only partially sampled, with ranges of $u v$ radii where no visibility information is available. However, even with this coarse sampling, the graphs show a decline in amplitude with $u v$ radius, indicating that our sources are spatially resolved. Simple 2D Gaussian fits to the maps presented in Fig. 3 also indicate 
Table 1. Summary of PdBI observations.

\begin{tabular}{|c|c|c|c|c|c|c|c|c|c|c|}
\hline \multirow[b]{2}{*}{ Name } & \multirow[b]{2}{*}{$\begin{array}{l}\text { Distance } \\
\text { (pc) }\end{array}$} & \multirow[b]{2}{*}{ Date } & \multicolumn{2}{|c|}{ Synthesized beam } & \multicolumn{2}{|c|}{ Peak position } & \multicolumn{2}{|c|}{ Fitted Gaussian } & \multirow[b]{2}{*}{$\begin{array}{c}v_{\mathrm{LSR}} \\
\left(\mathrm{km} \mathrm{s}^{-1}\right)\end{array}$} & \multirow[b]{2}{*}{$\begin{array}{c}\text { Flux } \\
\left(\mathrm{Jy} \mathrm{km} \mathrm{s}^{-1}\right.\end{array}$} \\
\hline & & & $\begin{array}{c}\text { FWHM } \\
\left({ }^{\prime \prime} \times{ }^{\prime \prime}\right)\end{array}$ & $\begin{array}{l}\text { PA } \\
\left({ }^{\circ}\right)\end{array}$ & $\begin{array}{c}\alpha_{2000} \\
\text { (h:m:s) }\end{array}$ & $\begin{array}{c}\delta_{2000} \\
\left({ }^{\circ}::^{\prime \prime}\right)\end{array}$ & $\begin{array}{c}\text { FWHM } \\
\left({ }^{\prime \prime} \times{ }^{\prime \prime}\right)\end{array}$ & $\begin{array}{l}\text { PA } \\
\left({ }^{\circ}\right)\end{array}$ & & \\
\hline V1057 Cyg & $\begin{array}{ll}g & 600\end{array}$ & 1993-Мay-31 & $7.02 \times 5.16$ & 2 & $20: 58: 53.8$ & $+44: 15: 28.7$ & $9.6 \times 5.2$ & 177 & 4.6 & 4.1 \\
\hline V1331 Cyg & 550 & 1993-Маy-31 & $7.30 \times 5.97$ & 147 & 21:01:09.2 & $+50: 21: 44.2$ & $9.7 \times 6.5$ & 148 & -0.1 & 2.5 \\
\hline V1515 Cyg & g 1000 & 1993-Мay-30 & $7.43 \times 6.90$ & 148 & $20: 23: 48.0$ & $+42: 12: 30.0$ & $21.9 \times 10.6$ & 166 & 5.1 & 5.1 \\
\hline V1735 Cyg & g 900 & 1993-May-30 & $8.01 \times 6.53$ & 133 & $21: 47: 20.5$ & $+47: 32: 06.3$ & $9.4 \times 9.4$ & 78 & 4.2 & 1.5 \\
\hline
\end{tabular}

Notes. Distances are from Sandell \& Weintraub (2001). The FWHM of the fitted Gaussians are deconvolved sizes.
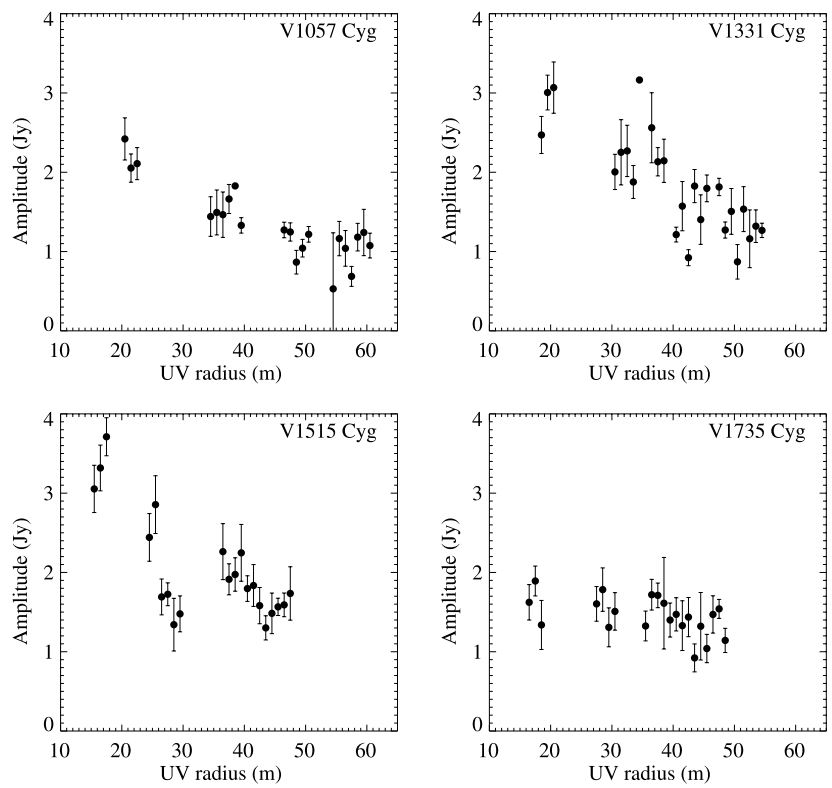

Fig. 2. Visibility amplitudes as a function of $u v$ radius. Data were binned in $1 \mathrm{~m}$-wide bins, and the error bars indicate the dispersion of data points within one bin.

that our targets are spatially resolved. The peak positions, deconvolved sizes, and PAs of the Gaussian fits are listed in Table 1. Gaussian models fitted to the visibilities give sizes within $\pm 2^{\prime \prime}$ and PAs within $\pm 10^{\circ}$ to those determined from the Gaussian fits to the images. Thus, we consider these numbers as representative uncertainties of the values given in Table 1. We emphasize that these fits are not physical envelope models, and thus the obtained parameters should be regarded only as rough quantitative estimates of the size of the emitting region.

V1057 Cyg and V1331 Cyg are the most compact sources in our sample, with FWHM of about $10^{\prime \prime} \times 5^{\prime \prime}$. Moreover, in both cases, the emission in centered precisely on the optical position of the stars. V1515 Cyg shows the most extended emission in our sample. The stellar position seems to be located at the southern tip of a bright, slightly curved, elongated filament, but extended emission can be seen both to the south and to the north of the $\operatorname{star}\left(\approx 20^{\prime \prime}\right.$ across $)$. A map produced for the channels around the emission line at $11.9 \mathrm{~km} \mathrm{~s}^{-1}$ reveals that this separate velocity component comes from a compact area $\approx 13^{\prime \prime}$ to the northwest $\left(\mathrm{PA} 330^{\circ}\right.$ ) of the optical source. The bulk of the emission in the V1735 Cyg region comes from an area with an FWHM of $\approx 9^{\prime \prime}$ centered 2'. 9 to the northwest with respect to the optical position of the star. Moreover, there is also some tentative extended emission towards the northwest about $14^{\prime \prime}$ from the stellar position.
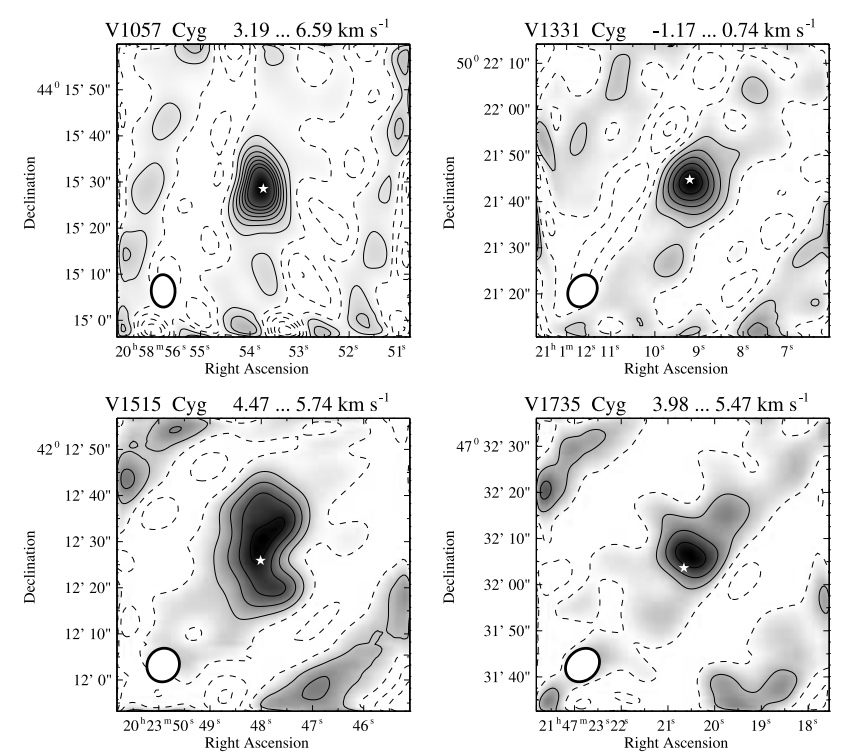

Fig. 3. ${ }^{13} \mathrm{CO}(1-0)$ maps of our targets, integrated in the velocity ranges indicated above the images. White asterisks indicate optical positions from SIMBAD. Beam sizes are $\approx 6 \times 7^{\prime \prime}$. The noise level is $\sigma=$ $0.10 \mathrm{Jy} \mathrm{km} \mathrm{s}^{-1}$; the solid contours are $2 \sigma, 4 \sigma, 6 \sigma, \ldots$; the dashed contours are $0,-2 \sigma,-4 \sigma$.

\subsection{Gas masses}

Following Scoville et al. (1986), the total $\mathrm{H}_{2}$ mass can be calculated from the observed ${ }^{13} \mathrm{CO}(1-0)$ line fluxes by

$M_{\mathrm{H}_{2}}=2.39 \times 10^{-9} \times \frac{\left(T_{X}+0.89\right)}{e^{-5.31 / T_{X}}} \frac{\tau_{13} \mathrm{CO}}{1-e^{-\tau}} \frac{D_{\mathrm{kpc}}^{2}}{X\left({ }^{13} \mathrm{CO}\right)} \int S_{\nu} \mathrm{d} v M_{\odot}$,

where $T_{X}$ is the excitation temperature, $\tau$ the optical depth, $D$ the distance of the source, $X\left({ }^{13} \mathrm{CO}\right)$ the molecular abundance relative to $\mathrm{H}_{2}$, and $\int S_{\nu} \mathrm{d} v$ the velocity-integrated line flux in units of Jy km s${ }^{-1}$. Using $T_{X}=50 \mathrm{~K}$ (as determined from dust continuum observations by Sandell \& Weintraub 2001), assuming the lines to be optically thin $(\tau \ll 1)$ and taking $X\left({ }^{13} \mathrm{CO}\right)=1.6 \times 10^{-6}$ (Langer \& Penzias 1993), the total gas masses are 0.12, 0.06, 0.43 , and $0.10 M_{\odot}$ for V1057 Cyg, V1331 Cyg, V1515 Cyg, and V1735 Cyg, respectively. Of course these numbers should be considered lower limits if the lines are actually optically thick, or if part of the ${ }^{13} \mathrm{CO}$ emission is resolved out by the interferometer. However, these values are in good agreement with total masses derived from single-dish $850 \mu \mathrm{m}$ dust continuum maps of comparable size by Sandell \& Weintraub (2001), which were $0.10,0.13,0.15$, and $0.42 M_{\odot}$ for V1057 Cyg, V1331 Cyg, V1515 Cyg, and V1735Cyg, respectively. This suggests that our interferometric observations probably recover most of the ${ }^{13} \mathrm{CO}$ emission. 


\section{4. $2.7 \mathrm{~mm}$ continuum}

Although the main focus of our data analysis is the study of the ${ }^{13} \mathrm{CO}$ emission, the PdBI observations could also be used to look for $2.7 \mathrm{~mm}$ continuum emission by excluding channels around the lines and collapsing the remaining channels. The resulting continuum maps have a typical $\mathrm{rms}$ noise of $2 \mathrm{mJy} / \mathrm{beam}$. We detected $2.7 \mathrm{~mm}$ continuum emission only for one of our targets, V1331 Cyg. This is not surprising because, based on the observations of Sandell \& Weintraub (2001), out of our four targets, V1331 Cyg is also the brightest at $1.3 \mathrm{~mm}$ and at $850 \mu \mathrm{m}$. The $2.7 \mathrm{~mm}$ emission we detected is not resolved, its position is consistent with the optical stellar position, and the measured total flux is $12 \pm 2 \mathrm{mJy}$. Assuming optically thin emission, dust opacity of $\kappa_{1.3 \mathrm{~mm}}=0.01 \mathrm{~cm}^{2} \mathrm{~g}^{-1}$ (Ossenkopf \& Henning 1994), an emissivity law of $\kappa \sim \lambda^{-1}$, and dust temperature of $50 \mathrm{~K}$ (Sandell \& Weintraub 2001), this flux corresponds to a total (gas+dust) mass of $0.19 M_{\odot}$, similar to what we obtained from the line flux of V1331 Cyg. Using $6 \mathrm{mJy}$ as a $3 \sigma$ upper limit for the $2.7 \mathrm{~mm}$ continuum flux of the other sources, we can give upper limits of $0.11,0.32$, and $0.26 M_{\odot}$ for V1057 Cyg, V1515 Cyg, and V1735 Cyg, respectively.

\section{Discussion}

In the following sections, we discuss what our interferometric ${ }^{13} \mathrm{CO}(1-0)$ data can add to what we already know about the circumstellar environment of our targets based on optical images, sub-millimeter continuum maps, CO line observations, and SED analysis from the literature. We also discuss whether the detected ${ }^{13} \mathrm{CO}$ emission can be associated with the envelopes.

\subsection{V1057 Cyg}

After its outburst in 1969-70, an eccentric ring-like nebulosity of $1^{\prime} \times 1.5$ appeared around V1057 Cyg (Herbig 1977). The monitoring of this ring in subsequent years indicated that it is fading together with the central star without any changes in the structure. This implies that the ring is a reflection nebula: a preexisting structure illuminated by the outbursting star, and not material ejected from the central source by the outburst. From the line wings of single-dish ${ }^{12} \mathrm{CO}$ data, Levreault (1988) and Evans et al. (1994) concluded the presence of a molecular outflow on a similar spatial scale. The surroundings of V1057 Cyg was mapped by Sandell \& Weintraub (2001), whose $850 \mu$ m continuum image indicates a rather compact, but resolved $\left(\approx 4^{\prime \prime}\right)$ source coinciding with the star and a fainter, north-south oriented filament.

In our ${ }^{13} \mathrm{CO}(1-0)$ data we do not detect emission either from the ring-like reflection nebula of from the north-south filament. However, our interferometric observations definitely resolve the central source: the deconvolved Gaussian FWHM at the distance of V1057 Cyg corresponds to about $5800 \times 3100$ AU. The lack of observable line wings, the relatively narrow line profile, and the compact spatial appearance of the emission points to quiescent gas, probably situated in the outer parts of a circumstellar envelope.

The infrared SED of the source (e.g. Ábrahám et al. 2004) suggests a continuous temperature distribution in the system. This supports our conclusion that there is an envelope associated with and heated by the central star. The presence and measured size of the envelope is consistent with the model prediction of $7000 \mathrm{AU}$ proposed by Green et al. (2006). They also suggest that the envelope has a rather large conical cavity, which explains the modest far-infrared excess. The relatively low extinction along the line-of-sight and the detectability of the $10 \mu \mathrm{m}$ silicate emission feature implies a close to pole-on geometry for the source, in accordance with its relatively symmetric shape in our interferometric observations. The circumstellar mass of $0.12 M_{\odot}$ derived from our ${ }^{13} \mathrm{CO}$ observations, consistently with the dust mass estimate by Sandell \& Weintraub (2001), is rather high, significantly exceeding the typical disk masses of T Tauri-type stars.

\subsection{V1331 Cyg}

V1331 Cyg is not an FUor in its present state, but - based on the similarity of its spectrum to that of V1057 Cyg prior to maximum light - is probably in a pre-outburst state, or between outbursts (McMuldroch et al. 1993). Single-dish and interferometric ${ }^{12} \mathrm{CO}$ and ${ }^{13} \mathrm{CO}$ observations by Levreault (1988) and McMuldroch et al. (1993) revealed a complex circumstellar environment containing a molecular outflow approximately along the line of sight, a flattened gaseous envelope of about $6000 \times 4400 \mathrm{AU}$ in size, and a gaseous expanding ring of about $41000 \times 28000 \mathrm{AU}$. The latter coincides well with the large ring-shaped optical reflection nebula seen by Quanz et al. (2007).

Consistently with the similar angular resolution measurements of McMuldroch et al. (1993), our interferometric ${ }^{13} \mathrm{CO}$ observations of V1331 Cyg reveal a rather compact structure towards the star with a deconvolved Gaussian FWHM of about $5300 \times 3600 \mathrm{AU}$ at the distance of V1331 Cyg. This core is also visible in continuum emission in the $850 \mu \mathrm{m}$ maps of Sandell \& Weintraub (2001), although it is only marginally resolved $\left(\lessgtr 6^{\prime \prime}\right.$ or $3300 \mathrm{AU})$. We suggest that both the ${ }^{13} \mathrm{CO}$ gas emission and the dust thermal emission originates in a circumstellar envelope, probably the same flattened structure that was proposed by McMuldroch et al. (1993) to explain the nature of the ${ }^{13} \mathrm{CO}$ emission. It is probable that the dust in this flattened envelope scatters the stellar light and gives rise to the inner ring observed in optical images by Quanz et al. (2007).

The presence of a circumstellar envelope around V1331 Cyg is also consistent with the SED, which exhibits significant infrared excess indicating substantial amounts of circumstellar material (e.g. Ábrahám et al. 2004). The geometry proposed by McMuldroch et al. (1993) and by Quanz et al. (2007) suggests that we see the inner part of the system through a conical cavity filled with a pole-on molecular outflow. However, we detect ${ }^{13} \mathrm{CO}$ emission neither from the outflow nor from the outer, large expanding ring, in agreement with the interferometric observations of McMuldroch et al. (1993).

\subsection{V1515Cyg}

V1551 Cyg brightened in optical light slowly during the 1940s and 1950s (Herbig 1977). Photographic plates from this time show a bright, narrow arc of nebulosity extending to the north and west of the star. Later images from the 1970s to the present day still show this northern arc, but a brighter nebulosity is also visible to the south and west, together forming a nearly complete circular ring with a diameter of $\approx 16^{\prime \prime}$ (corresponding to $16000 \mathrm{AU}$, see also the Sloan Digital Sky Survey ${ }^{2} r$-band image from 2003 in Fig. 4). That the size of this ring did not change significantly for 60 years but the brightness distribution did, suggests that it is a reflection nebula similar in nature to that around V1057 Cyg.

\footnotetext{
${ }^{2}$ http://www.sdss.org/
} 


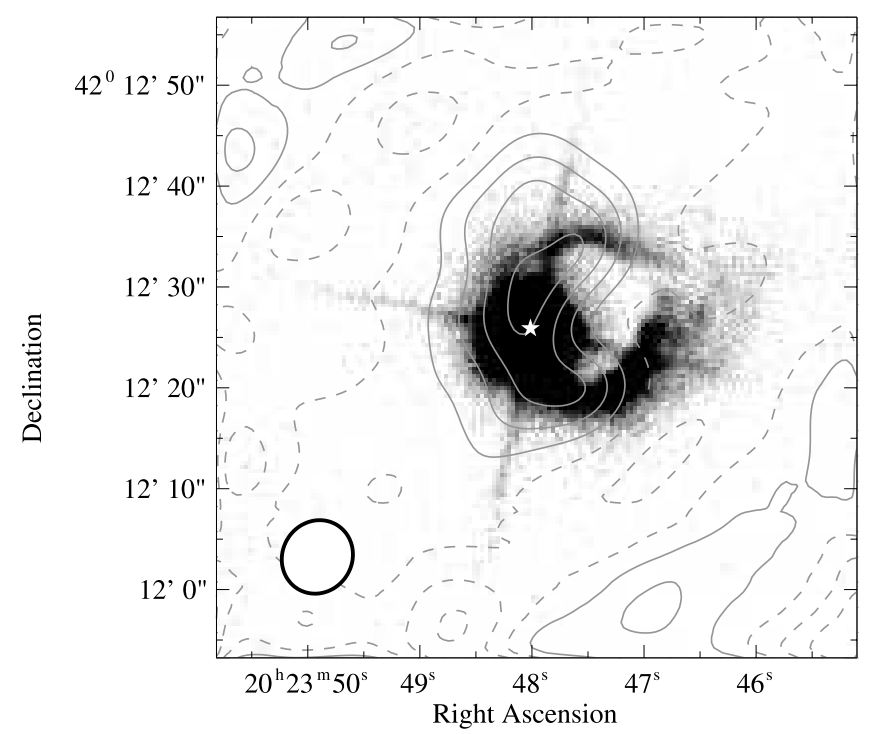

Fig. 4. The optical and millimeter environment of V1515 Cyg. The grayscale image in the background is an SDSS $r$-band image; the gray contours are the same ${ }^{13} \mathrm{CO}(1-0)$ contours as in Fig. 3. White asterisk indicate the optical stellar position.

Our ${ }^{13} \mathrm{CO}(1-0)$ measurement shows arc-shaped emission, which, plotted over the optical image in Fig. 4, clearly coincides with the ring-shaped reflection nebula. The similar morphology of the ${ }^{13} \mathrm{CO}$ emission and the scattered light, as well as the matching radial velocities of the ${ }^{13} \mathrm{CO}$ emission and the star suggest that the detected molecular gas is physically associated with the FUor. Similar to V1057 Cyg, the ${ }^{12} \mathrm{CO}$ line profile of V1515 Cyg in Evans et al. (1994) indicates a molecular outflow. Our ${ }^{13} \mathrm{CO}(1-0)$ data, however, show no high-velocity line wings; the emission line is narrow and thus probably traces quiescent, not outflowing material. We detect a small $\left(\lesssim 0.5 \mathrm{~km} \mathrm{~s}^{-1}\right)$ velocity difference between the northern and the southern parts of the ring, the southern part being slightly more redshifted. However, the ring is clearly not expanding.

A map of the $850 \mu \mathrm{m}$ dust continuum from Sandell \& Weintraub (2001) shows faint extended emission towards V1515 Cyg and also at about 10" to the north and northwest of the star. Although these observations were made with a relatively large $\left(15^{\prime \prime}\right)$ beam, the results are not inconsistent with a potentially arc-shaped dust emission. It is thus possible that the ring of material that is responsible for the optical reflection nebula and for the ${ }^{13} \mathrm{CO}$ emission also emits in dust continuum.

Although the observed molecular emission does not exactly peak towards the star, some dust and gas must be located in the vicinity of V1515 Cyg, forming a circumstellar envelope. This claim is suggested by the SED of the FUor, which was modeled with disk+envelope geometry by Turner et al. (1997) and Green et al. (2006). However, this envelope does not show up as a separate localized peak in our ${ }^{13} \mathrm{CO}$ map. We measured the highest gas mass towards this object within our sample, which can probably be explained by the contribution from both the circumstellar envelope and from the arc-shaped feature. Thus the $0.43 M_{\odot}$ is an upper limit for the envelope mass.

\subsection{V1735 Cyg}

V1735 Cyg had its outburst some time in the 1950s or the 1960s (Elias 1978). The star is associated with faint patches and filaments of reflected light within $1^{\prime}$. Submillimeter dust continuum observations by Sandell \& Weintraub (2001) indicate the presence of two sources: one associated with (albeit slightly offset from the optical position of) V1735 Cyg, and a brighter one located about 20-24" to the northeast (the deeply embedded Class I protostar V1735 Cyg SM1). Single-dish ${ }^{12} \mathrm{CO}$ and ${ }^{13} \mathrm{CO}$ observations (Levreault 1983; Richardson et al. 1985; Evans et al. 1994) have revealed a complex molecular gas structure in the vicinity of these sources. The ${ }^{12} \mathrm{CO}$ emission is extended on the arcminute spatial scale, and broad line wings indicate outflow activity. Evans et al. (1994) concluded that both V1735 Cyg and SM1 drive molecular outflows.

As for the other three FUors in our sample, our ${ }^{13} \mathrm{CO}$ interferometric observations of V1735 Cyg show narrow emission with no line wings. This again points to quiescent gas in some kind of envelope. Our map shows a compact peak close to V1735 Cyg, but with a definite offset of $2^{\prime \prime} .9$ to the northwest. Based on multifilter optical images, Goodrich (1987) discovered that V1735 Cyg itself is much more reddened than the surrounding reflection nebulosity. He speculates that the reason for this may be a dark cloud in front of V1735 Cyg. In this scenario, the dark cloud may be offset from the star, as long as it causes enough interstellar extinction to explain the observed colors. Based on the fact that the ${ }^{13} \mathrm{CO}$ emission we detect is offset from the stellar position, we propose that a significant part of the emission is coming from this foreground dark cloud. This suggestion is supported by the results of Quanz et al. (2007), who, based on the analysis of midinfrared ice features, conclude that the extinction for V1735 Cyg might be caused by ices somewhere in the line of sight to the source, rather than material related to the young star. The foreground structure, however, may not be completely unrelated to the star because of their identical radial velocities.

For continuum observations such as those presented in Weintraub et al. (1991) and in Sandell \& Weintraub (2001), the dominant source is not the FUor, but V1735 Cyg SM1. This source also emits molecular line emission, as shown by the single-dish ${ }^{12} \mathrm{CO}(3-2)$ map presented by Evans et al. (1994). Interestingly, we did not detect V1735 Cyg SM1 in ${ }^{13} \mathrm{CO}$. The reason for this may be partly that V1735 Cyg SM1 is separated from V1735 Cyg by about 20", thus it is at the very edge of our primary beam. Additionally, the submillimeter source is very extended, so the interferometer may filter out most of its emission.

\section{Summary and conclusions}

In this paper, we present interferometric observations of the ${ }^{13} \mathrm{CO}(1-0)$ line of four well known FUors, YSOs characterized by large optical outbursts due to enhanced disk accretion. For V1057 Cyg, V1515 Cyg, and V1735Cyg, these represent the first millimeter interferometric data published so far. This makes it possible to study the gas distribution on a few thousand AU spatial scale.

Although all of our sources are known to drive molecular outflows, as shown by the high-velocity line wings of ${ }^{12} \mathrm{CO}$, our data suggest that the ${ }^{13} \mathrm{CO}(1-0)$ emission traces quiescent gas. With the exception of V1515 Cyg, the size of the emitting region is within a few thousand $\mathrm{AU}$, consistent with typical circumstellar envelope sizes. Gas masses calculated from our ${ }^{13} \mathrm{CO}$ line fluxes and from dust continuum data from Sandell \& Weintraub (2001) are also consistent. This indicates that the ${ }^{13} \mathrm{CO}$ emission seen towards our sources mostly originates in a relatively compact circumstellar envelope, or, as in the case of V1735 Cyg, possibly in a small foreground cloud.

All of our sources are surrounded by reflection nebulosities, which are probably pre-existing structures illuminated by the brightened central source. With the exception of V1515 Cyg, we detect no ${ }^{13} \mathrm{CO}$ emission from these structures. For V1515 Cyg, 
the ${ }^{13} \mathrm{CO}$ emission coincides with the ring-shaped optical reflection nebula. This indicates that the source is surrounded by a ring of material that, on one hand, scatters the optical light of the central star, and on the other, emits at millimeter wavelengths. An important consequence is that attributing unresolved, singledish millimeter fluxes of V1515 Cyg to the circumstellar envelope may not be entirely correct.

Based on the appearance of the $10 \mu \mathrm{m}$ silicate feature, Quanz et al. (2007) define two categories of FUors. They argue that objects showing the feature in absorption (e.g. V1735 Cyg) are younger and still embedded in a circumstellar envelope. Objects showing the silicate band in emission (e.g. V1057 Cyg, V1331 Cyg, and V1515 Cyg) are more evolved, with a direct view on the surface layer of the accretion disk. However, in case of V1735 Cyg, the millimeter emission and the absorption at shorter wavelengths may be due to a foreground cloud, and consequently the object may be more evolved (less embedded) than it appears. Our ${ }^{13} \mathrm{CO}$ data, especially the case of V1515 Cyg and V1735 Cyg, demonstrate that millimeter emission and consequently absorption at other wavelengths may not be necessarily or exclusively associated with circumstellar envelopes. Thus, large beam, single-dish data alone are probably not enough to obtain a complete picture of the circumstellar environment of FUors. We suggest that any theory of the FUor phenomenon that interprets the geometry of the circumstellar material and its evolution using single-beam measurements must be checked and compared to interferometric observations in the future. Millimeter observations with facilities such as ALMA for both the dust continuum and for the line emission of molecules such as ${ }^{13} \mathrm{CO}, \mathrm{C}^{18} \mathrm{O}, \mathrm{HCO}^{+}$, and $\mathrm{HCN}$ will be a fruitful direction for future studies of FUors.

Acknowledgements. The author thanks Prof. Wolfgang J. Duschl for making the PdBI data available and Dr. Roberto Neri for his help during the data reduction. Discussions with Dr. Michiel Hogerheijde and Dr. Mária Kun greatly improved the presentation of the data analysis.

\section{References}

Ábrahám, P., Kóspál, Á., Csizmadia, S., et al. 2004, A\&A, 428, 89

Bechis, K. P., \& Lo, K. Y. 1975, ApJ, 201, 118

Brinch, C., Jørgensen, J. K., \& Hogerheijde, M. R. 2009, A\&A, 502, 199

Chavarría, C., Appenzeller, I., \& Bertout, C. 1979, A\&AS, 36, 465

Elias, J. H. 1978, ApJ, 223, 859

Evans, II, N. J., Balkum, S., Levreault, R. M., Hartmann, L., \& Kenyon, S. 1994, ApJ, 424, 793

Goodrich, R. W. 1987, PASP, 99, 116

Green, J. D., Hartmann, L., Calvet, N., et al. 2006, ApJ, 648, 1099

Hartmann, L., \& Kenyon, S. J. 1996, ARA\&A, 34, 207

Herbig, G. H. 1977, ApJ, 217, 693

Hogerheijde, M. R., van Dishoeck, E. F., Blake, G. A., \& van Langevelde, H. J. 1998, ApJ, 502, 315

Hogerheijde, M. R., van Dishoeck, E. F., Salverda, J. M., \& Blake, G. A. 1999, ApJ, 513, 350

Jørgensen, J. K., Hogerheijde, M. R., van Dishoeck, E. F., Blake, G. A., \& Schöier, F. L. 2004, A\&A, 413, 993

Kenyon, S. J., \& Hartmann, L. 1989, ApJ, 342, 1134

Langer, W. D., \& Penzias, A. A. 1993, ApJ, 408, 539

Levreault, R. M. 1983, ApJ, 265, 855

Levreault, R. M. 1988, ApJS, 67, 283

Matthews, B. C., Hogerheijde, M. R., Jørgensen, J. K., \& Bergin, E. A. 2006, ApJ, 652, 1374

McMuldroch, S., Sargent, A. I., \& Blake, G. A. 1993, AJ, 106, 2477

Momose, M., Ohashi, N., Kawabe, R., Nakano, T., \& Hayashi, M. 1998, ApJ, 504,314

Ossenkopf, V., \& Henning, T. 1994, A\&A, 291, 943

Quanz, S. P., Henning, T., Bouwman, J., et al. 2007, ApJ, 668, 359

Richardson, K. J., White, G. J., Avery, L. W., Lesurf, J. C. G., \& Harten, R. H. 1985, ApJ, 290, 637

Sandell, G., \& Weintraub, D. A. 2001, ApJS, 134, 115

Scoville, N. Z., Sargent, A. I., Sanders, D. B., et al. 1986, ApJ, 303, 416

Turner, N. J. J., Bodenheimer, P., \& Bell, K. R. 1997, ApJ, 480, 754

van Kempen, T. A., van Dishoeck, E. F., Salter, D. M., et al. 2009, A\&A, 498, 167

Vorobyov, E. I., \& Basu, S. 2006, ApJ, 650, 956

Weintraub, D. A., Sandell, G., \& Duncan, W. D. 1991, ApJ, 382, 270 